\title{
Three-dimensional discrete magnetic inversion using graph theory to determine the framework of isolated sources.
}

\author{
Romulo Rodrigues ${ }^{1}$, Edson Alonso Falla-Luza ${ }^{2}$, Rodrigo Bijani ${ }^{1}$ e Cosme Ponte-Neto ${ }^{2}$ \\ ${ }^{1}$ Universidade Federal Fluminense (UFF) \\ 2 Observatório Nacional (ON/MCTIC)
}

Copyright 2021, SBGf - Sociedade Brasileira de Geofísica.

This paper was prepared for presentation during the 17th International Congress of the Brazilian Geophysical Society, held in Rio de Janeiro, Brazil, 16 - 19 August, 2021.

Contents of this paper were reviewed by the Technical Committee of the 17th International Congress of The Brazilian Geophysical Society and do not necessarily represent any position of the SBGf, its officers or members. Electronic reproduction or storage of any part of this paper for commercial purposes without the written consent of the Brazilian Geophysical Society is prohibited.

\section{Abstract}

Applied geophysics is an important discipline for imaging and exploring the subsurface. Commonly, the geophysical data is acquired, treated and inverted to produce an interpreted model. In magnetics, a challenging aspect is obtain a reliable image of the causative magnetic sources, due to severe non uniqueness of magnetic inverse problems. To overcome this drawback, we revisit the work of Bijani et al. (2015) and use the equidistance function in the magnetic inverse problem of retrieving the framework of homogeneous and isolated sources. This is done by estimating the Cartesian coordinates of a set 0 magnetic dipoles, all with the same magnetization direction and dipole moment intensity. To achieve this goal in a stable way, the graph theory and the Minimum spanning tree problem are used to define the equidistance function. A preliminary test in a synthetic magnetic total-field anomaly produced by a vertical dike model is presented and briefly discussed. To bespeak the relevance of the equidistance function, we run the Genetic Algorithm for two values of regularizing parameters (i.e., $\lambda=0$ and $\lambda=5$ ). Preliminary results confirm the capability of the equidistance function in estimating a stable and compact ensemble of magnetic dipoles. Additionally, the magnetic properties are also recovered in a reliable way. Future perspectives are related to prepare more complex synthetic tests (i.e., the dipping dike model), a real data examples in a magnetic data acquired at Arraial do Cabo, Rio de Janeiro state. A more refined definition of the ideal regularizing parameter is also necessary.

\section{Introduction}

Magnetics is a valuable method for solving a variety of geophysical problems related to the knowledge of the subsurface. One of major concern of this method lies in the variations of magnetic properties of target sources beneath the Earth's surface. These rock units produce particular perturbations in the measured total field, reflecting variations of magnetic properties in the subsurface, including distribution of magnetic minerals and/or magnetization (Blakely 1996; Shearer 2005). The knowledge of these issues allow geophysicists to formulate different inverse problems for specific purposes. Commonly, imaging the subsurface by inversion of potential-field data is through mesh-based models (Ellis et al. 2012; Shearer, 2005; Paine et al., 2001; Uieda and Barbosa 2012; Bijani et al. 2017) A pioneer paper in using block model for solving a magnetic inverse problem is Li and Oldenburg (1996). In this work, the subsurface is represented by a mesh and the physical property (i.e., the magnetic susceptibility) within each cell is estimated through a gradient-descent method. In this set of problems, the magnetization direction is assumed to be induced by the local Geomagnetic field. To stabilize the ill-posed linear inverse problem, spatially dependent weighing functions are considered.

Another important magnetic inverse problem estimates the magnetization direction vector of geologic sources. For example, Lelièvre and Oldenburg (2009) estimate the Cartesian and spherical components of the total magnetization direction vector in each cell of a voxelized subsurface. To achieve good results, the method needs promising prior geologic information to be incorporated into the Cartesian and spherical formulation of the inverse problem. Following this guideline, Oliveira et al. 2015) invert the total-field anomaly to estimate the Cartesian components of the total magnetization vector in a leastsquares sense. Despite good results for synthetic tests, the major limitation of this method lies in the spherical approximation, which is not suitable for more complex geologic scenarios. More recently, Liu et al. (2018) propose a 3D inversion of magnetic data to deal with remanent magnetization and self-demagnetization of target sources by sequential estimates of both magnetic intensity and vector. To do so, the distortion analysis of Fedi et al. (1994) is considered and the Koenigsberger ratio is used to extract the remanent component of the total magnetization direction vector.

A third type of inverse problem that is totally applied to magnetic data is defining the approximate shape of causative sources. Particularly, a mesh-free interpretive model is necessary for a reasonable definition of the target source. In this case, a set of geometric parameters are estimated and the magnetization is known in advance Chakravarthi and Sundararajan (2007); Martins et al. (2011); Oliveira Jr and Barbosa (2013); Bijani et al. (2015 2017); Galley et al. (2020); Carter-McAuslan et al. (2015). In Bijani et al. (2015), a discrete gravity inverse problem is proposed. Additionally, a novel regularizing strategy (named equidistant function), based on the Minimum 
spanning tree problem, imposes homogeneity to estimated the 3-D distribution of a set of point masses. The inversion procedure is solve by means of an hierarchical genetic algorithm. Synthetic tests with a vertical and a dipping dike model are discussed for different values of the regularizing parameter.

Inverse problems to retrieve the shape of target sources are strongly dependent on initial models, specially when gradient-descent methods are used. Alternatively, practitioners are going for the global search optimization. In this set of techniques, also known as meta-heuristic methods, natural phenomena are numerically simulated, in such as simulated annealing (SA), ant colony optimization (ACO), and genetic algorithm (GA). The latter starts from a random population of candidate solutions, whom are progressively modified by the simulation of evolutionary behavior of biological systems, until an acceptable result is achieved Holland (1992); Chakraborty and Chaudhuri (2003); Deep et al. (2009); Montana (1994); Parker (1999). In this work, we revisit Bijani et al. (2015) and apply the equidistance function to a 3-D discrete magnetic inverse problem. In our case, the point masses are switch to magnetic dipoles and the total-field anomaly is now the data to be inverted. With this, we are intent to retrieve the magnetic framework of a target source. Additionally, the magnetic properties are also estimated during the generations of the implemented GA. To verify the relevance of our work, two tests with and without using the equidistance function are presented. In both cases, a vertical dike is the true source producing the noisecorrupted synthetic total field anomaly to be inverted.

\section{Methods}

\section{The discrete magnetic inverse problem}

Suppose that $\Delta \mathbf{T}^{o b s}$ is an $N$-dimensional vector containing the total-field anomaly (TFA), in nT, produced by a homogeneous source in subsurface, as shown in Figure 1 (a). The magnetic forward problem consists of representing the interpretive model by an ensemble of $M$ magnetic dipoles, all with the same magnetization direction and dipole moment intensity. The set of magnetic dipoles are placed in a three-dimensional domain, as can be seen in Figure 1](b).

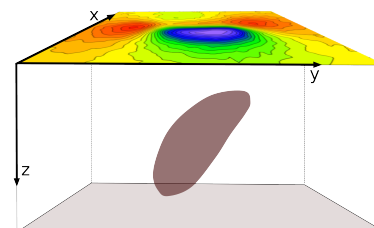

(a)

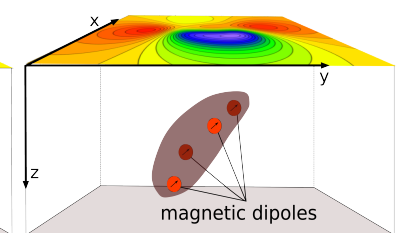

(b)
Figure 1: The magnetic problem sketch. (a) true homogeneous source, in brown, and the observed TFA at surface, represented by the contour map. (b) magnetic dipoles (orange circles), all with the same magnetization direction, and the predicted TFA in contour map at surface.

To calculate the $k$-th total-field anomaly produced by the set of magnetic dipole, we use Blakely (1996):

$$
\Delta T_{k}(\mathbf{q})=\left(\sum_{i=1}^{M} b_{x_{i}}+F_{x}\right)^{2}+\left(\sum_{i=1}^{M} b_{y_{i}}+F_{y}\right)^{2}+\left(\sum_{i=1}^{M} b_{z_{i}}+F_{z}\right)^{2}-|\mathbf{F}|
$$

where, $k$ goes from 1 to $N$ observation points, $F_{x}, F_{y}, F_{y}$ are the Cartesian components of the local Geomagnetic field, $|\mathbf{F}|=\left(F_{x}^{2}+F_{y}^{2}+F_{z}^{2}\right)^{1 / 2}$ represents the local Geomagnetic field intensity and $b_{x_{i}}, b_{y_{i}}, b_{z_{i}}$ are Cartesian components of the magnetic induction produced by the $i$-th dipole. The sum of equation 1 over all $N$ observation points creates the predicted data-vector $\Delta \mathbf{T}(\mathbf{q})$,referred here as predicted total-field anomaly.

Imagine know that $\mathbf{u}=\left(i, d, m_{0}\right)$ is a 3-dimensional vector containing the physical properties (i.e., magnetic inclination, declination and dipole moment intensity, respectively) of the magnetic dipoles. Additionally, vector $\mathbf{p}=\left(x_{1}^{\prime}, y_{1}^{\prime}, z_{1}^{\prime}, \ldots, x_{M}^{\prime}, y_{M}^{\prime}, z_{M}^{\prime}\right)$ is a $3 M$-dimensional vector with the Cartesian coordinates of the $M$ magnetic dipoles. Bunching vectors $\mathbf{u}$ and $\mathbf{p}$ ), we create a $3 M+3$ parametervector $\mathbf{q}$, which can be expressed in partitioned form as:

$$
\mathbf{q}=\left(\begin{array}{ll}
\mathbf{u} & \mathbf{p}
\end{array}\right)
$$

The discrete magnetic inverse problem consists of estimating a 3- $D$ spatial distribution of dipoles and their magnetic properties that minimizes the following data-misfit function in a least-squares sense:

$$
\phi(\mathbf{q})=\left\|\Delta \mathbf{T}^{o b s}-\Delta \mathbf{T}(\mathbf{m})\right\|^{2},
$$

where $\|$.$\| is the Euclidean norm and \Delta \mathbf{T}(\mathbf{q})$ is the predicted TFA computed by Equation 1 Equation 3 computes the difference between measured and predicted data at the same observation points. Commonly, geophysical inverse problem are ill-posed, which means that the information contained in the data is not sufficient to estimate the parameter-vector in a stable way. To deal with this issue, we define the following objective function:

$$
\Gamma(\mathbf{q})=\phi(\mathbf{q})+\lambda \theta(\mathbf{p}),
$$

where $\theta(\mathbf{p})$, also known as stabilizing function, imposes physical and/or geologic attributes to the solution. The non-negative scalar $\lambda$ controls a compromise between $\phi(\mathbf{m})$ and $\theta(\mathbf{p})$. Bijani et al. (2015) defined $\theta(\mathbf{p})$ as the equidistance function, whose purpose is impose homogeneity to the 3- $D$ spatial distribution of magnetic dipoles in subsurface. To do so, the ensemble o magnetic dipoles are treated as nodes of a graph and the Minimum Spanning Tree (MST) problem (Held and Karp 1971: Graham and Hell, 1985, Singh, 2009) is solved by Kruskal's algorithm (Kruskal 1956). The spacial distribution of magnetic dipoles should respect the MST concept, which is basically finding a fully-connected graph with minimum sum of edges. The equidistance function is defined by Bijani et al. (2015) as follows:

$$
\theta(\mathbf{p})=\frac{1}{M-1} \sum_{i=1}^{M-1}\left[\mathbf{d}_{i}^{M S T}(\mathbf{p})-\overline{\mathbf{d}}_{M S T}\right]^{2},
$$

where $\overline{\mathbf{d}}_{M S T}$ is the mean of distances of an MST, $\mathbf{d}_{i}^{M S T}(\mathbf{p})$ is a vector connecting adjacent dipoles in an MST, $M$ 
is the number of magnetic dipoles and $\mathbf{p}$ is the $M$ dimensional vector containing the Cartesian coordinates of the ensemble of dipoles. The minimization of Equation 5 ensure that all magnetic dipoles are equal distance apart. So, if $\theta(\mathbf{p})$ is zero, all edges of the MST have the same length.Once the discrete magnetic inverse problem is defined, we can go further and present the minimizing engine used in this work: the Genetic Algorithm.

\section{Inverse procedure: Genetic Algorithms}

Genetic algorithms consist of a random search algorithm based on the mechanics of natural selection and natural genetics (Goldberg and Holland, 1988, Montana, 1994). Genetic algorithms are widely used in optimization problems due to its capacity of finding the global minima of multi-modal functions (Holland, 1992).

Differently from deterministic methods, Genetic Algorithms (GAs) require not only a starting model, but a set of starting models, referred herein as an initial population. The initial population is randomly selected inside a search space, which is crucial for an accurate performance of the GA. In our magnetic inverse problem, the parameters to be estimated are: Cartesian coordinates of the ensemble of magnetic dipoles, magnetic inclination, magnetic declination and magnetic dipole moment intensity. Each parameter needs a search limit, which is initially defined by the interpreter. For a better estimation of the magnetic dipole moment intensity, the final estimated value should be as close as possible to the middle of initial search limit. Otherwise, we should redefine the ranges of the search limit and run the GA again. This is important aspect to ensure that the algorithm guides us to a promising minimum that fits the magnetic data.

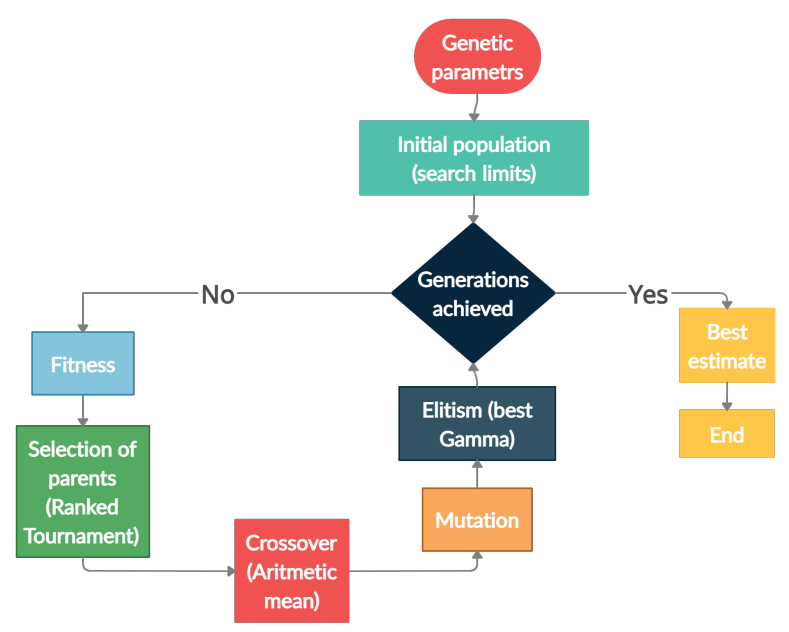

Figure 2: Flowchart of the our Hierarchical GA.

In this work we implement an Hierarchical Genetic Algorihtm (HGA), in python Language, and the flowchart of the code is presented in Figure 2. The relevant stages in the implementation of the genetic algorithm, such as crossover, mutation and selection of parents are widely discussed in Goldberg and Holland (1988) and Parker (1999).

The selection of parents operator simulates the how a family can be created. In our implementation, the Tournament is considered, which a promising individual is selected in a subgroup of the total population Goldberg and Holland 1988; Parker 1999).

A crossover operator consists of generating an offspring population based on the selected parents. A real-coded crossover based on arithmetic mean is implemented in our HGA (Goldberg and Holland, 1988; Bijani et al., 2015.

The Mutation operator is fundamental for preventing the estimates from local minimum entrapment Coello et al. (2004); Chakraborty and Chaudhuri (2003). In our case, each offspring population member (i.e., one set of magnetic dipoles) have a small probability of mutating one or more parameters (i.e., coordinates and/or magnetic parameters). The mutation is calculated by using a simple weighted arithmetic mean. The weights are randomly selected and define how close the parents and offsprings's parameters are.

We also consider an hierarchic procedure in our implementation. At this stage, a sub-population of $n_{e}$ individuals, decreasingly ordered in terms of the objective function $\Gamma(\mathbf{q})$ is replicated to the next generation. The hierarchic procedure accelerates the convergence, once promising solutions (i.e., with lower $\Gamma(\mathbf{q})$ values) are always placed into the current population (Chakraborty and Chaudhuri 2003).

\section{Tests with noise-corrupted synthetic data}

To produce the synthetic total-field anomaly, we buried a vertical prism with a true magnetic dipole moment intensity of $3.8 \times 10^{9} \mathrm{Am}^{2}$, elongated at $\mathrm{z}$ and $\mathrm{y}$-directions, ranging from $200 \mathrm{~m}$ to $4000 \mathrm{~m}$ and from $-2500 \mathrm{~m}$ to $2500 \mathrm{~m}$, respectively. The $x$ coordinate varies from $-500 \mathrm{~m}$ to $500 \mathrm{~m}$. The true magnetic inclination (I) and declination (D) of the vertical dike are both $0^{\circ}$, while the regional magnetic inclination $\left(I^{\prime}\right)$ and magnetic declination $\left(D^{\prime}\right)$ are $5^{\circ}$ and $70^{\circ}$, respectively. The forward calculations are based on (Blakely, 1996). Two tests with different regularizing parameters are presented, similarly with Bijani et al. (2015). Additionally, the data size is $N=400$ and the total-field anomaly is corrupted with random Gaussian noise of zero mean and a standard deviation of $5 \mathrm{nT}$. For both tests presented in this work, The search limit values for each parameter are highlighted in Table 1 .

Table 1: The search limits use in the initial population. In the lines of the table are found minimum and maximum values of each parameter, respectively. In the columns observed the parameters are $\mathrm{x}, \mathrm{y}$, and $\mathrm{z}$ coordinate, magnetic inclination, magnetic declination, and magnetic dipole moment intensity.

\begin{tabular}{|c|c|c|c|c|c|}
\hline $\mathrm{X}(\mathrm{m})$ & $\mathrm{Y}(\mathrm{m})$ & $\mathrm{Z}(\mathrm{m})$ & $I^{\prime}\left({ }^{\circ}\right)$ & $D^{\prime}\left({ }^{\circ}\right)$ & Moment $\left(\mathrm{Am}^{2}\right)$ \\
\hline$-5000 \mathrm{~m}$ & -5000 & 200 & -5 & -5 & $1.24 \times 10^{9}$ \\
\hline $5000 \mathrm{~m}$ & 5000 & 4000 & 5 & 5 & $1.29 \times 10^{9}$ \\
\hline
\end{tabular}

\section{Test with $\lambda=0$}

In this case, we are not making use of the equidistance function during the minimization of $\Gamma(\mathbf{q})$. Figure 3 (a) and (c) show different spatial perspectives of the best initial solution, while Figure 3 (b) and (b) show the best 
solution of the last generation. As expected, the best initial solution presents a high dispersion of magnetic dipoles, while the best solution after 3000 generations shows the dipoles occupying the significant part of the vertical dike. Despite evidences of a good result, we do not guarantee that the dipoles are homogeneously distribution along the interpretive model. (a)

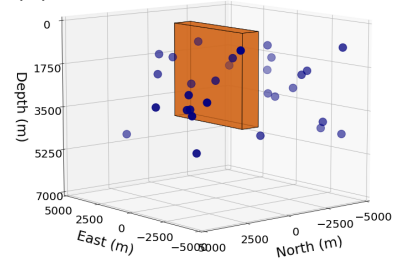

(c)

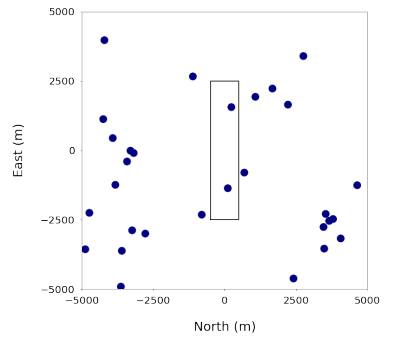

(b)

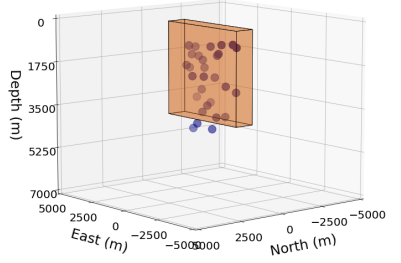

(d)

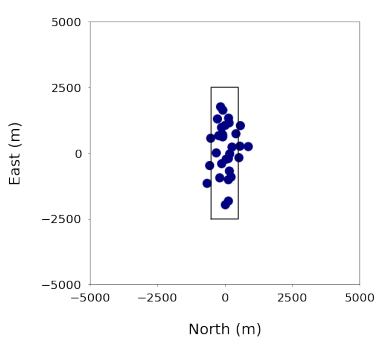

Figure 3: The results of the HGA for $\lambda=0$. Orange prism represent the vertical dike and blue spheres symbolize the magnetic dipoles. (a) Best initial solution. (b) Best final solution after 3000 generations (3D view). (c) Best initial solution (2D view). (d) Best final solution after 3000 generations (2D view).

Figure 4 (a) presents the observed total-field anomaly produced by the vertical dike model.

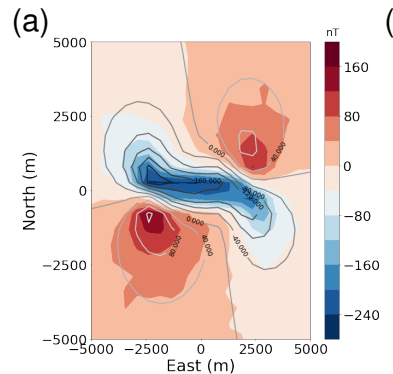

(c)

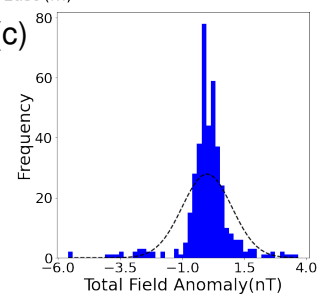

(b)

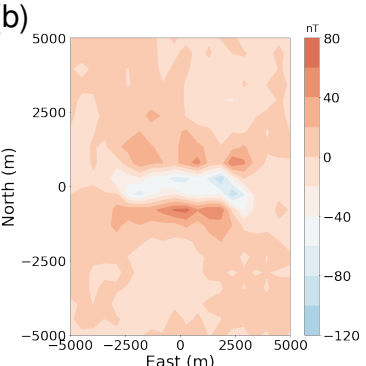

$\mu=0.36584$ $\sigma=18.27629$

Figure 4: Geophysics results, obtained through HGA with $\lambda$ equal 5. (a) Fit between observed (contour map) and predicted total-field anomaly (gray contour lines). Residual map. (c) Residuals histogram.

Additionally, the residuals are represented in contour map (The figure $4 \mathrm{~b}$ ) and in histogram $4 \mathrm{k}$ ). These images show that the predicted total-field anomaly reasonably fits the observed data. Despite, we reinforce that the solutions are not stabilized.Figure 5 (a) shows the convergence of the objective function (i.e., 4) over the generations. We clearly observe a fast convergence but with some stagnancy after generation 1000. Figures (5 (b) and 5 (c) show the magnetic inclination and declination through the generations of our HGA, respectively. The true direction is quite close the the estimated one, despite of no stabilization.

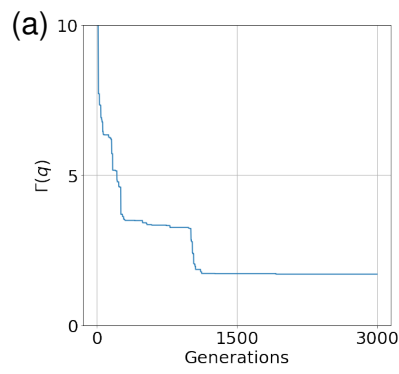

(b)

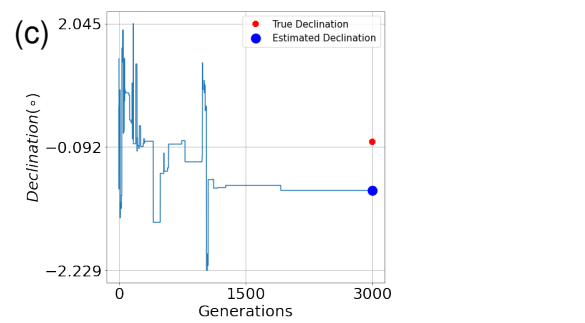

Figure 5: Convergence graphs with $\operatorname{com} \lambda$ equal 0 . The red circle represents the true magnetic inclination while the blue circle represent the estimated magnetic inclination. (a) Convergence of the objective function. (b) Magnetic inclination estimates over the generations of our HGA. (c) Magnetic declination estimates during the HGA generations.

\section{Vertical dike with $\lambda=5$}

In this test, we turn on the equidistance function and set $\lambda=5$ by trial. Figure 3 (a) and 6 (c) show different spatial perspectives of the best initial solution, while the final solution is shown in Figure 3 (b) and (d). Although we can see a good distribution of magnetic dipoles in Figure 6 (b), the 2D view of 6(d) shows that the set of magnetic dipoles are more compact, which confirm the stabilization offered by the equidistance function. 
(a)

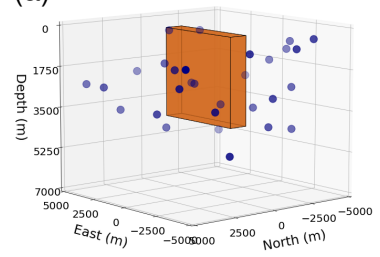

(b)

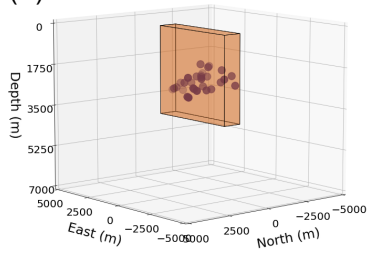

(d)
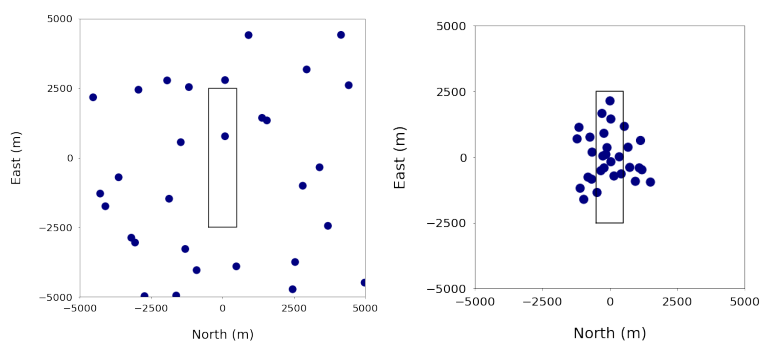

Figure 6: Results of our HGA for $\lambda=5$. Orange prism represent the vertical dike model and blue spheres are the ensemble of magnetic dipoles. (a) Best initial solution. (b) Best final solution after 3000 generations (3D view). (c) Best initial solution (2D view) and (d) best final solution after 3000 generations (2D view).

Figure 7 (a) shows the observed and predicted total-field anomaly for this test. In this case, the fit is not desired, which might be related to an exaggerated $\lambda$ value. The residual map and histogram presented in Figure 7 (b) and (c) respectively reinforce the undesired misfit. (a)

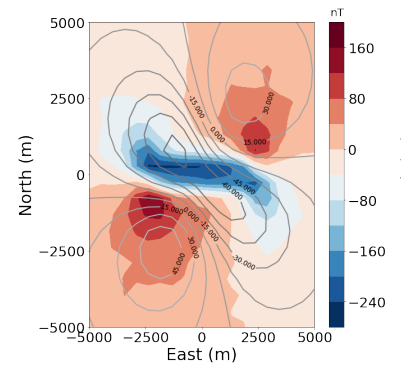

(c)

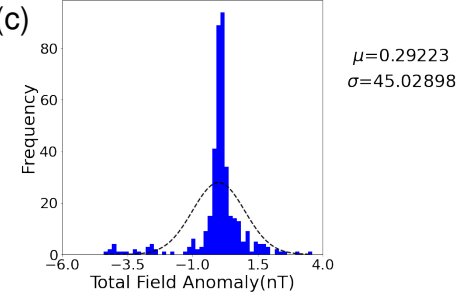

Figure 7: Results for $\lambda=5$. (a) Fit between observed (contour map) and predicted (contour gray lines) total-field anomaly . (b) Residual map. (c) Residuals histogram.

Figure 8 (b) and (c) shows the behavior of the datamisfit function $(\phi((q)))$ and the equidistance function $(\theta(\mathbf{p}))$ separated. We observe that the HGA prioritize the minimization of $(\theta(\mathbf{p}))$, which is clearly related to the high value of $\lambda$.

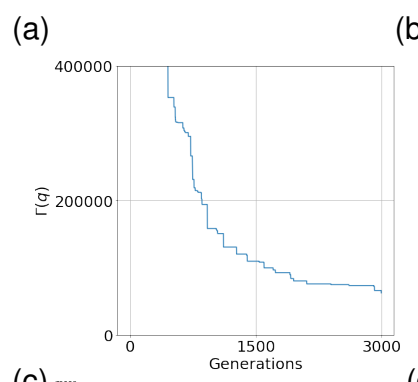

(b)

(c)

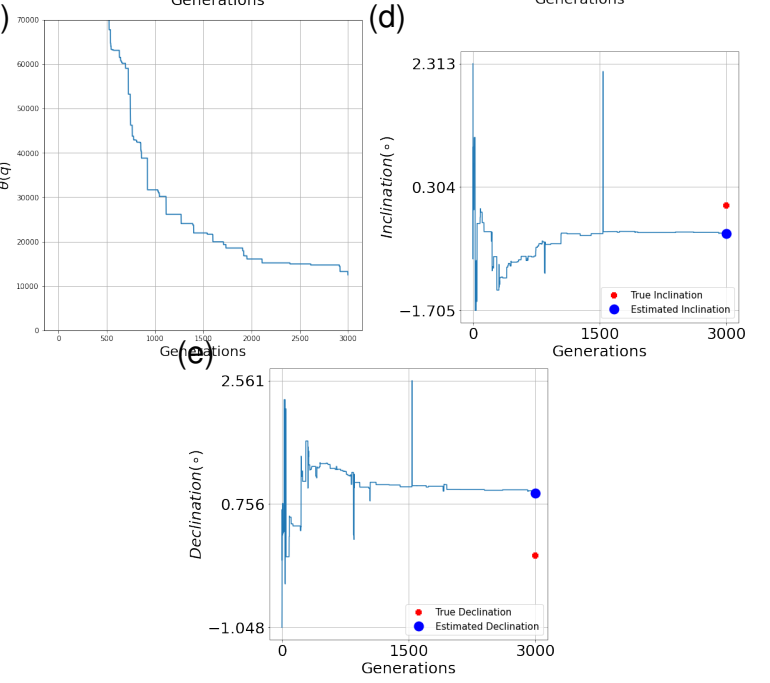

Figure 8: Convergence curves for $\lambda=5$. (a) Objective function. (b) Data-misfit function. (c) Equidistance function. (d) magnetic inclination and (e) magnetic declination.

Figure 8 (a) show the convergence curve of the objective function, stagnated once again. Figures 8 (d) and 8 (e) present the magnetic inclination and declination convergence, respectively. We observe a similar behavior of the first test, which indicates no need for stabilizing this set of parameters. In this case, we include the convergence curve of the equidistance function $\theta(\mathbf{q})$, following the same guideline in Bijani et al. (2015).

\section{Conclusions}

We hope that through this work we have demonstrated that the equidistance function, previously presented in a gravity inverse problem, also produces reliable results for a three-dimensional magnetic inverse problem. Using a set of dipoles with identical magnetic properties, we implement a Hierarchical genetic algorithm to minimize an objective function composed of a data-misfit term and a stabilizing term. The latter is a dispersion function that measures the compactness of the ensemble of dipoles. Preliminary test on a vertical dike model suggests improvements related to the search for an ideal regularizing parameter. Additionally, convergence curves show that the HGA converges really fast, but some stagnancy is also observed and need to be treated accurately. Improvements in specific genetic operators, as crossover and selection of parents are required. Another synthetic test comprising a dipping magnetic dike and also a real magnetic data example, acquired in Arraial do Cabo, RJ are also considered as future investigations. 


\section{Acknowledgments}

First author thanks CAPES for scholarship. Authors are grateful to developers of python libraries, once this work is fully implemented in this programming language.

\section{References}

Bijani, R., P. G. Lelièvre, C. F. Ponte-Neto, and C. G. Farquharson, 2017, Physical-property-, lithology-and surface-geometry-based joint inversion using pareto multi-objective global optimization: Geophysical Journal International, 209, 730-748.

Bijani, R., C. F. Ponte-Neto, D. U. Carlos, and F. J. Silva Dias, 2015, Three-dimensional gravity inversion using graph theory to delineate the skeleton of homogeneous sources: Geophysics, 80, G53-G66.

Blakely, R. J., 1996, Potential theory in gravity and magnetic applications: Cambridge University Press.

Carter-McAuslan, A., P. G. Lelièvre, and C. G. Farquharson, 2015, A study of fuzzy c-means coupling for joint inversion, using seismic tomography and gravity data test scenarios: Geophysics, 80, W1-W15.

Chakraborty, B., and P. Chaudhuri, 2003, On the use of genetic algorithm with elitism in robust and nonparametric multivariate analysis: Austrian Journal of Statistics, 32, 13-27.

Chakravarthi, V., and N. Sundararajan, 2007, 3d gravity inversion of basement relief:a depth-dependent density approach: Geophysics, 72, 123-132.

Coello, C., G. Pulido, and M. Lechuga, 2004, Handling multiple objectives with particle swarm optimization: IEEE Transactions on Evolutionary Computation, 8, 256-279.

Deep, K., K. P. Singh, M. L. Kansal, and C. Mohan, 2009, A real coded genetic algorithm for solving integer and mixed integer optimization problems: Applied Mathematics and Computation, 212, 505-518.

Ellis, R., B. de Wet, and I. Macleod, 2012, Inversion of magnetic data for remanent and induced sources, in ASEG Extended Abstracts 2012: 22nd Geophysical Conference: Australian Society of Exploration Geophysicists (ASEG), ASEG Extended Abstracts, $1-4$.

Fedi, M., G. Florio, and A. Rapolla, 1994, A method to estimate the total magnetization direction from a distortion analysis of magnetic anomalies: Geophysical Prospecting, 42, 261-274.

Galley, C. G., P. G. Lelièvre, and C. G. Farquharson, 2020, Geophysical inversion for $3 d$ contact surface geometry: Geophysics, 85, K27-K45.

Goldberg, D. E., and J. H. Holland, 1988, Genetic Algorithms and machine learning: Machine Learning, 3, 95-99.

Graham, R. L., and P. Hell, 1985, On the history of the minimum spanning tree problem: IEEE Ann. Hist. Comput., 7, 43-57.

Held, M., and R. M. Karp, 1971, The travelingsalesman problem and minimum spanning trees: Part II: Mathematical Programming, 1, 6-25.

Holland, J. H., 1992, Adaptation in natural and artificial systems: an introductory analysis with applications to biology, control, and artificial intelligence: MIT Press.

Kruskal, Jr., J. B., 1956, On the shortest spanning subtree of a graph and the traveling salesman problem: Proceedings of the American Mathematical Society, 7,
48-50. (ArticleType: research-article / Full publication date: Feb., 1956 / Copyright Â@ 1956 American Mathematical Society).

Lelièvre, P. G., and D. W. Oldenburg, 2009, A 3D total magnetization inversion applicable when significant, complicated remanence is present: Geophysics, 74, L21-L30.

Li, Y., and D. Oldenburg, 1996, 3-d inversion of magnetic data: GEOPHYSICS, 61, 394-408.

Liu, S., M. Fedi, X. Hu, Y. Ou, J. Baniamerian, B. Zuo, Y. Liu, and R. Zhu, 2018, Three-dimensional inversion of magnetic data in the simultaneous presence of significant remanent magnetization and self-demagnetization: example from Daye iron-ore deposit, Hubei province, China: Geophysical Journal International, 215, 614-634.

Martins, C. M., W. A. Lima, V. C. Barbosa, and J. B. Silva, 2011, Total variation regularization for depth-tobasement estimate: Part 1 - mathematical details and applications: Geophysics, 76, I1-I12.

Montana, D. J., 1994, Strongly typed genetic programming: Evolutionary Computation, 3, 199-230.

Oliveira, V. C., D. P. Sales, V. C. F. Barbosa, and L. Uieda, 2015, Estimation of the total magnetization direction of approximately spherical bodies: Nonlinear Processes in Geophysics, 22, 215-232.

Oliveira Jr, V. C., and V. C. Barbosa, 2013, 3-d radial gravity gradient inversion: Geophysical Journal International, 195, 883-902.

Paine, J., M. Haederle, and M. Flis, 2001, Using transformed TMI data to invert for remanently magnetised bodies: Exploration Geophysics, 32, 238-242.

Parker, P. B., 1999, Genetic algorithms and their use in geophysical problems: $\mathrm{PhD}$ thesis, University of California, Berkeley.

Shearer, S. E., 2005, Three-dimensional inversion of magnetic data in the presence of remanent magnetization: PhD thesis, Citeseer.

Singh, A., 2009, An artificial bee colony algorithm for the leaf-constrained minimum spanning tree problem: Applied Soft Computing, 9, 625-631.

Uieda, L., and V. C. F. Barbosa, 2012, Robust 3d gravity gradient inversion by planting anomalous densities: Geophysics, 77, G55. 